Georgetown University Law Center

Scholarship @ GEORGETOWN LAW

2018

Reaching for Mediocrity: Competition and Stagnation in Pharmaceutical Innovation

Son Le

Doctoral Student, Department of Economics, Georgetown University, snl9@georgetown.edu

Neel U. Sukhatme

Georgetown University Law Center, nus6@georgetown.edu

This paper can be downloaded free of charge from:

https://scholarship.law.georgetown.edu/facpub/2152

This open-access article is brought to you by the Georgetown Law Library. Posted with permission of the author. Follow this and additional works at: https://scholarship.law.georgetown.edu/facpub

Part of the Health Law and Policy Commons, and the Intellectual Property Law Commons 


\title{
Reaching for Mediocrity: Competition and Stagnation in Pharmaceutical Innovation
}

\author{
Son Le* and Neel U. Sukhatme**
}

October 15, 2018

\begin{abstract}
Patents might incentivize invention but they do not guarantee firms will invest in projects that maximize social utility. We model how risk-neutral firms' ability to obtain substantial private returns on marginal new technologies causes them to "reach for mediocrity" by investing in socially-suboptimal projects, even in the presence of competition and new entrants. Focusing primarily on pharmaceutical innovation, we analyze various policy interventions to solve this underinvestment problem. In particular, we describe a new approach to patents - a value-based patent system, which ties patent protection to the underlying invention's social value - and show how it incentivizes socially-optimal innovation.
\end{abstract}

\footnotetext{
*Doctoral student, Dept. of Economics, Georgetown University - snl9@georgetown.edu

**Associate Professor, Georgetown University Law Center; Affiliated Faculty, Georgetown McCourt School of Public Policy - neel.sukhatme@law.georgetown.edu, 202-662-4035

The authors thank participants at the 2017 Works-in-Progress IP Colloquium, the 2017 IP Scholars Conference, and the Georgetown Law Faculty Workshop, and Axel Anderson, Scott Baker, M. Gregg Bloche, Dan
} 


\section{Contents}

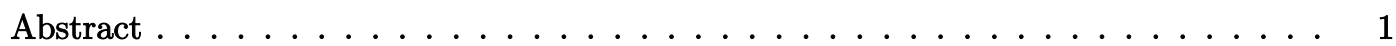

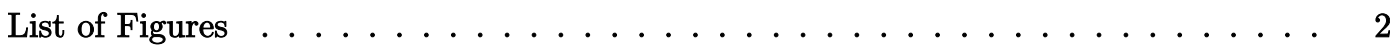

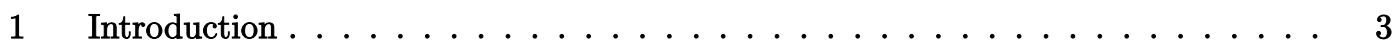

2 Prior Literature and Empirical Motivation . . . . . . . . . . . . . . . . . . 4

$3 \quad$ Discrete Choice Model . . . . . . . . . . . . . . . . . . . . . . . . 7

$3.1 \quad$ Monopolistic environment . . . . . . . . . . . . 7

$3.2 \quad$ Competitive environment $\ldots \ldots \ldots \ldots \ldots$

$3.3 \quad$ New entrants . . . . . . . . . . . . . . . . . . . . . 12

4 Continuous Choice Model . . . . . . . . . . . . . . . . . . . . . 13

$5 \quad$ Policy Interventions $\ldots \ldots \ldots \ldots \ldots \ldots \ldots$

$5.1 \quad$ Comparative Statics . . . . . . . . . . . . . . . . . 17

5.2 Increase in Technology Threshold . . . . . . . . . . . . . . . . . 21

$5.3 \quad$ Rewards and Taxes . . . . . . . . . . . . . . . . . 21

$5.4 \quad$ Value-Based Patent Protection . . . . . . . . . . . . . . . . 24

$5.5 \quad$ Policy under Incomplete Information . . . . . . . . . . . . . . . . 25

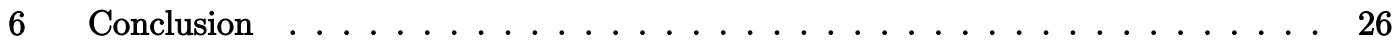

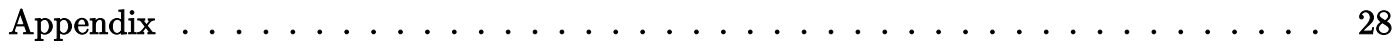

A Proof of Proposition $5.6 \ldots \ldots \ldots \ldots \ldots \ldots$

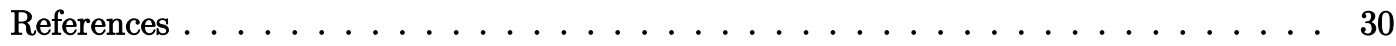

\section{List of Figures}

1 Metastatic Colorectal Cancer Drugs: Increments in Average Overall Survival v.

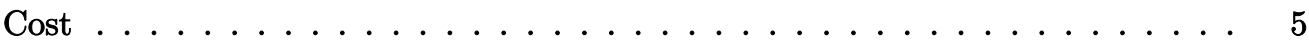

Cao, Brian Galle, David Hyman, Scott Kostyshak, Joshua Krieger, Joshua Mitts, Larry Samuelson, and Josh Teitelbaum. Special thanks to Leo Acklin, Yinan Lin, and Flora Feng for excellent research assistance. All errors are the authors' own. 


\section{Introduction}

In a competitive market, research and development is risky because firms must recover up-front fixed costs through lower future expenses or increased future sales. Unfortunately, R\&D is likely produced in socially-suboptimal quantities due to the knowledge spillovers it generates and the potential for competitors to free-ride on originators' efforts. Accordingly, society subsidizes R\&D through direct subsidies and research grants, tax credits and deductions, rewards, and patents (Grabowski et al. [2015]; Hemel and Ouellette [2013]; Shavell and Van Ypersele [2001]; Kremer [1998]).

While many scholars have focused on which innovation policies optimally encourage R\&D spending, there has been less focus on the specific $R \& D$ activities these firms pursue. Put differently, firms typically have some choice in their R\&D investments. Given this choice, are they picking socially-optimal R\&D projects?

Generally, the profit motive should induce firms to choose R\&D projects that society values highly. But as we show in this paper, sometimes firms are excessively conservative compared to a social planner when selecting investment projects in innovation markets. The problem is particularly acute in pharmaceuticals, though our argument could also extend to other markets.

The following section provides empirical motivation for the paper and explains why firms often invest in marginally beneficial or "me-too" drugs, which are often highly profitable despite providing minimal marginal social benefits. We then present both discrete- and continuouschoice models that explain how a firm's return function - what the firm earns as a function of an underlying invention's social value - creates a wedge between its incentives and those of the social planner. We also show how this wedge can persist even with competition and potential new entrants into the market.

We then assess how various conventional policy interventions - such as changing the drug approval threshold, awarding prizes, and assessing lump-sum taxes - might address this disconnect between social and private incentives. Among other things, we show that if the threshold to award a prize is set too low, prizes can result in even lower quality innovation than would occur in their absence. 
Finally, we model a novel approach to patents - a value-based patent system, where more socially-valuable inventions receive heightened patent protection (e.g., longer patent duration) as compared to less socially-valuable ones. We show how a value-based patent system generates higher-quality innovation than the status quo, while not inadvertently incentivizing low-value innovation, as would occur with across-the-board increases in patent protection. We close with a few suggestions on how a value-based patent system might be implemented in practice, referencing other research we are conducting contemporaneously.

\section{Prior Literature and Empirical Motivation}

Several scholars have recognized problems with marginal or me-too innovation in pharmaceuticals. Jayadev and Stiglitz [2009] claim superior innovative drugs can become unprofitable when drug companies use better marketing on inferior me-too products. Fojo et al. [2014] similarly argue that follow-on products can crowd out innovation on new therapeutic targets. ${ }^{1}$

Me-too drugs are not rare. Régnier [2013] notes that of the 431 new molecular entities approved by the U.S. Food and Drug Administration (FDA) between 1990 and 2004, 58\% had "therapeutic qualities similar to those of one or more already marketed." Similarly, Fojo et al. [2014] observe that out of 71 drugs approved by the FDA between 2002 and 2014 for solid tumors, "the median gains in progression-free and overall survival (OS) were a very modest 2.5 and 2.1 months, respectively." And under standards suggested by the American Society of Clinical Oncology (ASCO) Cancer Research Committee, only $42 \%$ of these 71 drugs would constitute "clinically meaningful improvements."

Worse, most marginal drugs are not cheap. Figure 1 illustrates the underlying problem. It summarizes the results for all completed Phase 3 interventional clinical trials with statistically significant results for treatment of metastatic colorectal cancer (mCRC), as reported at www.clinicaltrials.gov. The $\mathrm{x}$-axis shows the difference in median overall survival times for treatment groups as compared to control groups, averaged across all trials for each drug. The y-axis lists the annual cost of treatment for each drug, estimated using data collected from

\footnotetext{
${ }^{1}$ In contrast, DiMasi and Paquette [2005] suggest most follow-on drugs are developed at the same time as pioneering drugs. They claim such drugs are not harmful and actually increase therapeutic choice.
} 
goodRx.com, an online retailer of prescription medication. Averaging across these studies, each additional month of overall survival costs about $\$ 56,000$ per year of treatment (and most mCRC patients undergo multiple years of treatment). Certainly an individual might value one additional month of life at $\$ 56,000$ or more. But from a social welfare standpoint, this value greatly exceeds any reasonable estimate of the average value of an additional month of human life, especially given the relatively low quality of life for patients with mCRC. See, e.g., Lee et al. [2009] (estimating the value of a quality-adjusted year of life for patients on dialysis to be about $\$ 129,000)$. The price charged does not come close to the value delivered. ${ }^{2}$

Figure 1: Metastatic Colorectal Cancer Drugs: Increments in Average Overall Survival v. Cost

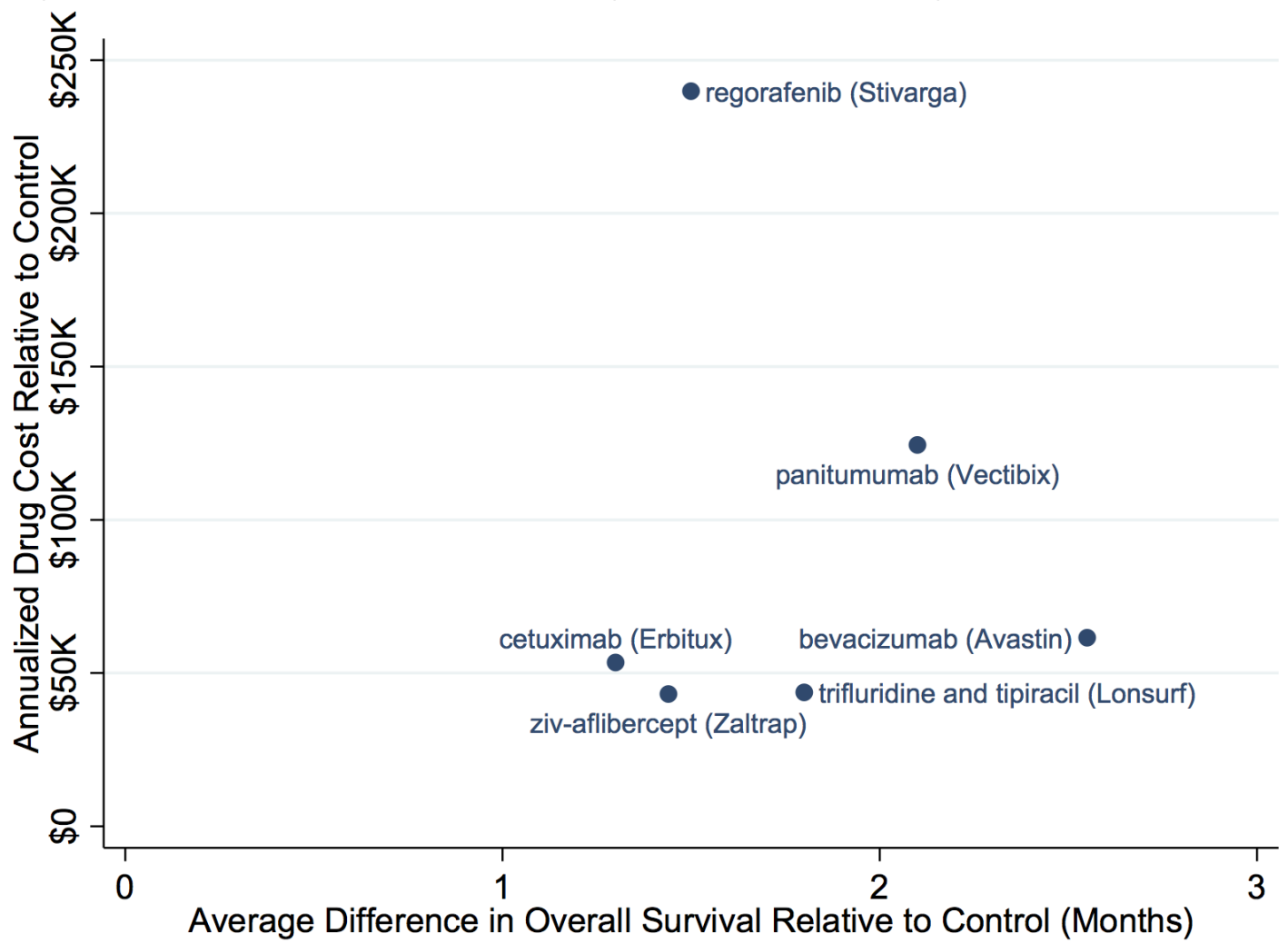

Sources: Overall survival data from clinicaltrials.gov. Cost data estimated using prices on goodRx.com as of September 2016.

\footnotetext{
${ }^{2}$ Some might wonder if the small difference in median outcomes obscures larger benefits that the treatment might bestow on certain subpopulations. While this might be possible, it is not reflected in the clinical trial data. Rather, when one examines Kaplan-Meier curves, which allow one to compare how survival rates vary on a patent-by-patient basis between treatment and control groups, it is clear the two groups remain relatively close in terms of overall performance throughout the duration of these studies.
} 
Why is there this disconnect between the value delivered by pharmaceutical innovation and the prices charged by innovators? In related work, Sukhatme and Bloche [2018] explore this phenomenon, highlighting the impact of moral hazard in health insurance markets, a singleminded focus of regulators and market participants on statistical versus therapeutic significance, and drug makers' marketing strategies for follow-on drugs. Most importantly, they highlight the psychological and political economy factors that make it difficult for payers and regulators to limit access to technologies that marginally improve outcomes at tremendous cost. Specifically. once a new medical technology becomes available, a tangle of human psychology, cultural mores, clinical ethics and stakeholder expectations makes it difficult for payers and regulators to refuse to pay for such treatment, even if its cost greatly exceeds the benefits it provides. So firms can earn large private benefits for new medical technologies that improve upon existing treatments, however barely. Since path-breaking, high-value technologies are often riskier to pursue than marginally beneficial technologies, firms are skewed toward pursuing inventions that are less socially valuable. Put differently, the various distortions in pharmaceutical markets that allow firms to earn outsized private benefits for marginal technologies also induce those firms to produce such technologies rather than riskier, more socially-valuable therapies.

While other scholars do not appear to have tackled this particular question, they have modeled somewhat related phenomena. Most notably, Ganuza et al. [2009] model how priceinelastic doctors, who make drug purchase decisions on behalf of insured patients, induce firms to invest in follow-on innovations instead of pioneering drugs and encourage them to spend on persuasive as compared to informative marketing to increase demand for me-too drugs. Focusing on diseases such as HIV/AIDS, Leoni and Sandroni [2016] model how excessive patent duration can encourage firms to collude with one another to produce marginal innovations rather than full cures. González et al. [2016] generate a model of follow-on drugs with patients that are horizontally and vertically differentiated, and intermediate doctors that act as perfect agents for the health care system. Antoñanzas et al. [2011] model a game in which pharmaceutical firms launch product line extensions after their drug patents expire, with "loyal" doctors prescribing the product line extension and "non-loyal" ones taking into account drug prices. Finally, Bardey et al. [2010] model a dynamic game with firms, consumers, and regulatory agencies to measure 
how reference pricing - which involves categorizing drugs by class and determining a common price for that class - can change firms' dynamic innovation incentives and reduce health.

Our paper builds on this previous literature by adding a new dimension: the risk/return tradeoff that technology firms face when deciding which investment project to pursue. For pharmaceuticals, the choice is often between pursuing a follow-on innovation (which might focus on a known molecular target that has already been successfully targeted by an existing drug) or pursuing a potentially more beneficial but riskier original innovation. Our model formalizes and explores the consequences of what recent empirical research by Krieger et al. [2018] shows: that pharmaceutical companies view incremental innovation as less valuable but much less risky than more novel, potentially path-breaking innovations.

While we have not found other models of such decisionmaking, other scholars have looked more generally at how risk and return influence firms' investment decisions. Notably, RoseAckerman [1991] explored how the risk of bankruptcy can cause firm decisionmakers to sacrifice expected returns for lower risk.

\section{Discrete Choice Model}

\subsection{Monopolistic environment}

We begin with a simple discrete choice model to illustrate why firms might pursue sociallysuboptimal innovation. A risk-neutral representative firm seeks to create a new innovation in a particular field. ${ }^{3}$ In this monopolistic environment, we assume the inventor owns a patent on the new technology and hence has a legal monopoly over its production.

Let $X$ be the realized net social value of the new technology. In other words, it is the value of the new technology to a social planner. ${ }^{4}$ Each technology has a threshold, $\underline{X}$, and any technologies whose social value $X<\underline{X}$ are normalized and assumed to be worth 0 for the inventor. For simplicity, we assume that technologies with $X<\underline{X}$ have no social value either,

\footnotetext{
${ }^{3}$ In the pharmaceutical context, one can view the underlying field as a particular drug indication (e.g., first-line treatment for metastatic colorectal cancer).

${ }^{4}$ All private and social values referenced here can either be in aggregate or per capita terms; the intuition of the model remains the same either way.
} 
though our results remain the same if we assume the social planner receives some value $x$ for these inventions.

In some markets, $\underline{X}$ might be interpreted as the most advanced technology that exists, or the "state of the art." In other markets, one can think of $\underline{X}$ as the threshold below which a technology provides the firm with no profits. For pharmaceuticals, the latter interpretation might be more appropriate, where $\underline{X}$ is the baseline safety/efficacy profile below which the FDA will not approve a drug, and hence the drug generates no private value.

The returns the inventor receives are not necessarily the same as the social planner. Let $m(X)$ be the inventor's profit as a function of the social value of the new technology. Suppose $m(X)$ takes the following piecewise linear form:

$$
m(X)=\left\{\begin{array}{ll}
0 & \text { for } X<\underline{X} \\
X+b, & \text { for } X \geq \underline{X}
\end{array}, \text { in which } b \geq 0\right.
$$

The value $b$ is the guaranteed fixed private return the inventor receives if his technology meets or exceeds the threshold $\underline{X}$. In the context of pharmaceuticals, $b$ is the private benefit firms receive for producing drugs that barely improve over existing therapies (Sukhatme and Bloche [2018]). In other contexts, $b$ might be the private cost of producing a new product inferior to the current state of the art, such as private costs incurred on firm managers and board members if a product fails and the firm goes bankrupt. When $b=0$, there is no distortion and the inventor's profit function is the same as the social planner; hence the inventor will choose socially-optimal projects.

The realized social value of each potential technology the inventor could select is uncertain. It is represented by a binomial distribution in which with probability $p$ the technology achieves $\bar{X}>\underline{X}$ and with probability $1-p$ it falls below $\underline{X}$. Assume there are only two choices for the inventor: a risky project summarized by $\left(p_{H}, \bar{X}_{H}\right)$ and a safe one $\left(p_{L}, \bar{X}_{L}\right)$ in which $\bar{X}_{H}>$ 
$\bar{X}_{L}>\underline{X}$ and $0<p_{H}<p_{L}<1$. Suppose further that $p_{H} \bar{X}_{H}>p_{L} \bar{X}_{L}$, which implies choosing the risky project is socially optimal. ${ }^{5}$

The inventor will pick the project with higher expected private return. Therefore, he will pick the safe project iff:

$$
\begin{aligned}
\left(1-p_{L}\right) * 0+p_{L} m & \left(\bar{X}_{L}\right)>\left(1-p_{H}\right) * 0+p_{H} m\left(\bar{X}_{H}\right) \\
& \Longleftrightarrow p_{L}\left(\bar{X}_{L}+b\right)>p_{H}\left(\bar{X}_{H}+b\right) \\
& \Longleftrightarrow b>\frac{p_{H} \bar{X}_{H}-p_{L} \bar{X}_{L}}{p_{L}-p_{H}} \equiv b_{M}>0
\end{aligned}
$$

If the distortion $b$ exceeds the threshold $b_{M}$, the inventor will pursue the less risky, sociallysuboptimal project. Intuitively, this is because the profit differential between high- and low-value inventions is not as large for the inventor as it is for the social planner. While an inventor still receives a higher return if she picks a risky project and the resulting value exceeds $\underline{X}$, she has to trade off that increase in return with the heightened risk of falling below $\underline{X}$ and earning nothing. Unlike the social planner, the inventor obtains a fixed benefit $b$ from any innovation that improves on the status quo, however marginally. Since the inventor receives this "boost" unconditionally for any invention whose social value exceeds $\underline{X}$, and higher-value inventions are riskier, the inventor might choose a less risky project than the social planner.

\subsection{Competitive environment}

Next we introduce competition into the environment. First, assume there are two identical firms that seek to create new innovations in a particular field, with both firms having the same return function $m(X)$ as before. Both are entitled to produce the underlying technology (i.e., neither can exclude the other from production via a patent). Again we assume there are only two choices for the firms, a risky project $\left(p_{H}, \bar{X}_{H}\right)$ and a safe one $\left(p_{L}, \bar{X}_{L}\right)$. We have three cases (due to firms being identical), with the corresponding return matrices specified below:

\footnotetext{
${ }^{5}$ Here we make the standard assumption that a social planner is risk-neutral and seeks to maximize expected value. We recognize our analysis might yield a different result under other less common approaches, such as those used in regret theory models in which policymakers choose policies that minimize maximum regret.
} 
Case 1. Both firms pick the safe projects:

\begin{tabular}{c|c|c|}
\multicolumn{1}{c}{$<\underline{X}$} & $\bar{X}_{L}$ \\
\cline { 2 - 3 }$<\underline{X}$ & 0,0 & $\bar{X}_{L}+b, 0$ \\
\cline { 2 - 3 } $\bar{X}_{L}$ & $0, \bar{X}_{L}+b$ & $\frac{\bar{X}_{L}}{2}+b, \frac{\bar{X}_{L}}{2}+b$ \\
\cline { 2 - 3 } & &
\end{tabular}

Case 2. Both firms pick the risky projects:

\begin{tabular}{c|c|c|}
\multicolumn{1}{c}{} & \multicolumn{1}{c}{$<\underline{X}$} & $\bar{X}_{H}$ \\
\cline { 2 - 3 }$<\underline{X}$ & 0,0 & $\bar{X}_{H}+b, 0$ \\
\cline { 2 - 3 } $\bar{X}_{H}$ & $0, \bar{X}_{H}+b$ & $\frac{\bar{X}_{H}}{2}+b, \frac{\bar{X}_{H}}{2}+b$ \\
\cline { 2 - 3 } & &
\end{tabular}

Case 3. One firm picks the risky and the other firm picks the safe project:

\begin{tabular}{c|c|c|}
\multicolumn{1}{c}{$<\underline{X}$} & $\bar{X}_{L}$ \\
\cline { 2 - 3 }$<\underline{X}$ & 0,0 & $\bar{X}_{L}+b, 0$ \\
\cline { 2 - 3 } $\bar{X}_{H}$ & $0, \bar{X}_{H}+b$ & $\bar{X}_{H}+b, 0$ \\
\cline { 2 - 3 } & &
\end{tabular}

In other words, the two firms split $\bar{X}_{L}\left(\bar{X}_{H}\right)$ if they both pick the low-value (high-value) project and both are successful, and a firm receives nothing if its competitor achieves a higher-value outcome than it does.

Note that in our model, $b$ is not whittled down by competition. This is a key assumption that reflects our underlying view of $b$ - it is a private return that firms receive that is independent of the value they deliver, so long as their product exceeds the technology threshold $\underline{X}$. Whether viewed as being driven by patient psychology and political economy (Sukhatme and Bloche [2018]) or private costs borne by firm managers if a project fails (Rose-Ackerman [1991]), competition does not diminish $b$ here. Our approach is supported by some empirical evidence in the pharmaceutical context, which suggests that introduction of new me-drugs might not lower other related drugs' prices. See Figure 1; also compare Ekelund and Persson [2003] (introduction of branded substitutes has little effect on introductory price or price dynamics in Swedish market) with Lu and Comanor [1998] (more branded substitutes in U.S. market lead to lower 
introductory prices and lower subsequent price increases). At any rate, even if $b$ is reduced in the real world by competition, the distortion we identify here is possible so long as $b$ remains sufficiently large, as discussed below.

We look for the condition in which both firms will pick the socially-suboptimal, safe project in a Nash equilibrium. Conditioning on the other firm picking the safe project, the expected return for a firm if it also picks the safe project is:

$$
\begin{array}{r}
p_{L}^{2}\left(\frac{\bar{X}_{L}}{2}+b\right)+\left(1-p_{L}\right) p_{L}\left(\bar{X}_{L}+b\right) \\
\quad=p_{L}\left[\frac{\bar{X}_{L}}{2} p_{L}+\left(1-p_{L}\right) \bar{X}_{L}+b\right]
\end{array}
$$

If it picks the risky project, the expected return will be:

$$
p_{H}\left(\bar{X}_{H}+b\right)
$$

Therefore, the best response for the firm will be the safe project iff:

$$
\begin{aligned}
& p_{L}\left[\frac{\bar{X}_{L}}{2} p_{L}+\left(1-p_{L}\right) \bar{X}_{L}+b\right]>p_{H}\left(\bar{X}_{H}+b\right) \\
\Longleftrightarrow & b>\frac{p_{H} \bar{X}_{H}-p_{L}\left[\frac{\bar{X}_{L}}{2} p_{L}+\left(1-p_{L}\right) \bar{X}_{L}\right]}{p_{L}-p_{H}} \equiv b_{O}
\end{aligned}
$$

Notice that $b_{O}>b_{M}$, which means adding in a competitor raises the threshold over which a high $b$ will result in a distortion. Still, duopoly only mitigates, and does not eliminate, the potential problem. If the private distortion $b$ is large enough, both firms will still choose the socially-suboptimal project.

More generally, we can expand our analysis to $n$ identical competing firms. If $n-1$ firms choose the low-value project, the remaining firm will also choose the low-value project if the expected return from doing so exceeds the expected return from choosing the high-value project:

$$
p_{L} \sum_{k=0}^{n-1}\left(\left(\begin{array}{c}
n-1 \\
k
\end{array}\right) p_{L}^{k}\left(1-p_{L}\right)^{(n-1-k)}\right)\left(\frac{\bar{X}_{L}}{k+1}+b\right)>p_{H}\left(\bar{X}_{H}+b\right)
$$


Multiplying out the left-hand side, we can simplify the first term on that side as follows:

$$
\begin{array}{r}
p_{L} \bar{X}_{L} \sum_{k=0}^{n-1}\left(\frac{(n-1) !}{k !(n-1-k) !} p_{L}^{k}\left(1-p_{L}\right)^{(n-1-k)} \frac{1}{k+1}\right) \\
=\left(\frac{p_{L} \bar{X}_{L}}{n}\right) \sum_{k=0}^{n-1}\left(\frac{n !}{(k+1) !(n-(k+1)) !} p_{L}^{k}\left(1-p_{L}\right)^{(n-1-k)}\right) \\
=\left(\frac{p_{L} \bar{X}_{L}}{n}\right) \sum_{j=1}^{n}\left(\frac{n !}{j !(n-j) !} p_{L}^{j-1}\left(1-p_{L}\right)^{(n-j)}\right) \\
=\left(\frac{\bar{X}_{L}}{n}\right)\left(\sum_{j=0}^{n}\left(\left(\begin{array}{c}
n \\
j
\end{array}\right) p_{L}^{j}\left(1-p_{L}\right)^{(n-j)}\right)-\left(1-p_{L}\right)^{n}\right) \\
=\left(\frac{\bar{X}_{L}}{n}\right)\left(1-\left(1-p_{L}\right)^{n}\right)
\end{array}
$$

And so the whole equation simplifies to the following condition:

$$
b>\frac{p_{H} \bar{X}_{H}-\left[1-\left(1-p_{L}\right)^{n}\right] \frac{\bar{X}_{L}}{n}}{p_{L}-p_{H}} \equiv b_{n}
$$

As $n \rightarrow \infty$, the second term in the numerator goes to 0 , and so:

$$
b>\frac{p_{H} \bar{X}_{H}}{p_{L}-p_{H}} \equiv b_{C}
$$

Accordingly, even in a perfectly competitive market, if the private distortion $b$ exceeds the threshold $b_{C}$, all firms in the market will still choose the socially-suboptimal project. So no amount of competition guarantees that firms will choose the social optimum.

\subsection{New entrants}

Finally, we consider an environment in which a monopolistic incumbent seeks to create a new innovation while simultaneously facing the threat of competition from a potential entrant. The entrant chooses between: no entry, entry and pick the safe project, or entry and pick the risky 
project. The entry cost is $c>0$. Assume further that $p_{H} \bar{X}_{H}>c$, or the expected social value of the risky project is higher than the entry cost. The return matrix of the game is:

\begin{tabular}{c|c|c|}
\multicolumn{1}{c}{ No entry } & \multicolumn{1}{c}{ Risky } & Safe \\
\cline { 2 - 3 } Entry \& Risky & $p_{H}\left(\bar{X}_{H}+b\right), 0$ & $p_{L}\left(\bar{X}_{L}+b\right), 0$ \\
\cline { 2 - 3 } & $p_{H}^{2}\left(\frac{\bar{X}_{H}}{2}+b\right)+$ & \\
$p_{H}\left(1-p_{H}\right)\left(\bar{X}_{H}+b\right)$, & $p_{L}\left(1-p_{H}\right)\left(\bar{X}_{L}+b\right)$, \\
Entry \& Safe & $p_{H}^{2}\left(\frac{\bar{X}_{H}}{2}+b\right)+$ & $p_{H}\left(\bar{X}_{H}+b\right)-c$ \\
\cline { 2 - 3 } & $p_{H}\left(1-p_{H}\right)\left(\bar{X}_{H}+b\right)-c$ & \\
\cline { 2 - 3 } & $p_{H}\left(\bar{X}_{H}+b\right)$, & $p_{L}^{2}\left(\frac{\bar{X}_{L}}{2}+b\right)+$ \\
& $p_{L}\left(1-p_{H}\right)\left(\bar{X}_{L}+b\right)-c$ & $p_{L}^{2}\left(\frac{\bar{X}_{L}}{2}+b\right)+$ \\
& & $p_{L}\left(1-p_{L}\right)\left(\bar{X}_{L}+b\right)-c$ \\
& &
\end{tabular}

The Nash equilibrium of this game will be (Safe, Entry \& Safe) if $b>b_{O}$. So if the distortion exceeds the threshold $b_{O}$, both the entrant and the incumbent will pick the suboptimal safe choice, as was the case in the duopolistic environment.

\section{Continuous Choice Model}

Now we generalize the previous model into one in which a firm can choose from a continuous set of potential R\&D projects, with continuous returns. As before, $X$ is the social value of the new technology with a technology threshold, $\underline{X}$, below which the technology has zero private value. The planner always receives return $X$ from the invention, whether it exceeds the technology threshold $\underline{X}$ or not.

The inventor's profit function $m(X)$ now takes the following piecewise linear form:

$$
m(X)= \begin{cases}0 & \text { for } X<\underline{X} \\ m X+b, & \text { for } X \geq \underline{X}, 0<m \leq 1, b \geq 0\end{cases}
$$

As before, $b$ is the guaranteed fixed private return the inventor receives if her technology meets or exceeds the technology threshold $\underline{X} . m$ is the return factor the inventor receives depending on how much her invention's social value exceeds this threshold. 
Once again, the inventor knows his profit function but not the realized outcome. ${ }^{6}$ The social value of each potential technology the inventor could select is represented by a distribution $G\left(X ; \mu, \sigma^{2}\right)$ over its social return. Assume that within the inventor's choice set, $G$ is fully identified by its mean $\mu$ and variance $\sigma^{2}$ and that $G\left(X ; \mu, \sigma^{2}\right)$ is fully differentiable with respect to $\mu$ and $\sigma^{2}$.

Furthermore, as $\mu$ increases, so does $\sigma^{2}$. That is $\sigma^{2} \geq h(\mu)$, where $h^{\prime}(\mu) \geq 0$, so social value and risk increase together.

Proposition 4.1. (The planner's solution) The planner chooses the project with the highest mean $\mu$. He is indifferent with respect to variance $\sigma^{2}$.

Proof. This follows immediately from the planner's risk-neutrality; he does not care about the variance of the distribution $G(X)$ and only picks projects with the highest mean $\mu$, since his return is just $X$.

By contrast, the inventor chooses a technology with mean $\mu$ and variance $\sigma^{2}$ that maximizes her expected return $E(m(X))$. This can be written as the following optimization problem:

$$
\begin{array}{r}
\max _{\mu, \sigma^{2}} E\left[m(X) \mid \mu, \sigma^{2}\right] \\
\text { s.t. } \\
\sigma^{2} \geq h(\mu)
\end{array}
$$

To begin, consider the scenario in which the inventor picks the lowest variance project for a given $\mu$. In other words, the constraint in the optimization problem binds and $\sigma^{2}=h(\mu)$. The optimization problem then becomes:

\footnotetext{
${ }^{6}$ We could easily add uncertainty to the inventor's knowledge of her profit function by adding some symmetrical noise (e.g., $\epsilon \sim N\left(0, \sigma_{\epsilon}^{2}\right)$ ) to the value of $m$. But this would not change the results here since the inventor is an expected utility maximizer, $m$ only enters the optimization problem linearly, and $E(m+\epsilon)=m$.
} 


$$
\begin{array}{r}
\max _{\mu} E[m(X) \mid \mu, h(\mu)] \\
=\max _{\mu} \int_{\underline{X}}(m x+b) d G(x ; \mu, h(\mu)) \\
\Longleftrightarrow \max _{\mu} \int_{\underline{X}}\left(x+\frac{b}{m}\right) d G(x ; \mu, h(\mu)) \\
=\max _{\mu}\left(\underline{X}+\frac{b}{m}\right)[1-G(\underline{X} ; \mu, h(\mu))]+\int_{\underline{X}}^{\infty}(x-\underline{X}) d G(x ; \mu, h(\mu))
\end{array}
$$

First-order condition (FOC):

$$
\begin{array}{r}
\left.=\left(\underline{X}+\frac{b}{m}\right) \frac{d}{d \mu}[1-G(\underline{X} ; \mu, h(\mu))]+\frac{d}{d \mu} \int_{\underline{X}}^{\infty}(x-\underline{X}) d G(X) \mid \mu, h(\mu)\right] \\
=-\left(\underline{X}+\frac{b}{m}\right) G_{2}(\underline{X} ; \mu, h(\mu)) h^{\prime}(\mu)-\left(\underline{X}+\frac{b}{m}\right) G_{1}(\underline{X} ; \mu, h(\mu)) \\
+\frac{d}{d \mu} \int_{\underline{X}}^{\infty}(x-\underline{X}) d G(x ; \mu, h(\mu))
\end{array}
$$

As $\mu$ increases, both $\mu$ and $h(\mu)$ go up. The first term in FOC, - $\left(\underline{X}+\frac{b}{m}\right) G_{2}(\underline{X} ; \mu, h(\mu)) h^{\prime}(\mu)$, is the partial effect of an increase in variance $h(\mu)$ on $G(\underline{X} ; \mu, h(\mu))$. It reflects the chance of falling below the threshold $\underline{X}$.

The second term $-\left(\underline{X}+\frac{b}{m}\right) G_{1}(\underline{X} ; \mu, h(\mu))$ is the partial benefit of higher $\mu$ on $G(\underline{X} ; \mu, h(\mu))$. Since increasing $\mu$ while keeping $\sigma^{2}$ constant is equivalent to a first-order stochastic dominant shift, it reduces the chance of falling below the threshold $\underline{X}$. Thus, $G_{1}(\underline{X} ; \mu, h(\mu)) \leq 0$ and $-\left(\underline{X}+\frac{b}{m}\right) G_{1}(\underline{X} ; \mu, h(\mu)) \geq 0$.

The third term $\frac{d}{d \mu} \int_{\underline{X}}^{\infty}(x-\underline{X}) d G(x ; \mu, h(\mu))>0$ measures the benefit of a higher $\mu$ on the added reward of obtaining an invention above the threshold, which is an increasing function of $\mu$.

We can see, therefore, that a sufficient condition for the firm to pick a lower $\mu$ than the social planner is: 


\section{Condition 1.}

$$
\begin{array}{r}
\left(\underline{X}+\frac{b}{m}\right) G_{2}(\underline{X} ; \mu, h(\mu)) h^{\prime}(\mu) \geq \\
-\left(\underline{X}+\frac{b}{m}\right) G_{1}(\underline{X} ; \mu, h(\mu))+\frac{d}{d \mu} \int_{\underline{X}}^{\infty}(x-\underline{X}) d G(x ; \mu, h(\mu))
\end{array}
$$

Proposition 4.2. If the inventor picks the lowest variance project $\sigma^{2}$ for a given $\mu$, and Condition 1 is satisfied, then the inventor chooses $\mu_{M}$ that is strictly lower than that picked by the planner, $\mu_{S}$.

Proof. Follows directly from the discussion above.

Note that Condition 1 can also be written in terms of $h^{\prime}(\mu)$ :

$$
h^{\prime}(\mu) \geq \frac{-\left(\underline{X}+\frac{b}{m}\right) G_{1}(\underline{X} ; \mu, h(\mu))+\frac{d}{d \mu} \int_{\underline{X}}^{\infty}(x-\underline{X}) d G(x ; \mu, h(\mu))}{\left(\underline{X}+\frac{b}{m}\right) G_{2}(\underline{X} ; \mu, h(\mu))}
$$

Written this way, it becomes clear that Condition 1 essentially requires risk to increase with return steeply enough such that it exceeds a certain threshold. Then, the inventor will choose a socially-suboptimal project.

We now make the following assumption:

Assumption 1. (Diminishing return to risk) $\frac{\partial^{2}}{\partial\left(\sigma^{2}\right)^{2}} E\left[m(X) \mid \mu, \sigma^{2}\right] \leq 0$

Assumption 1 requires the return to taking more risks at a given level of $\mu$ is decreasing with $\sigma^{2}$. This assumption reflects the reality that there are diminishing gains from taking on more risk for a distribution with a given $\mu$.

With Assumption 1 in place, we can now show the following:

Proposition 4.3. If Condition 1 and Assumption 1 hold, then the inventor will pick the lowest $\sigma^{2}$ for any given $\mu$, as well as a socially-suboptimal project. That is, the constraint $\sigma^{2} \geq h(\mu)$ will bind, and the inventor's choice $\mu_{M}<\mu_{S}$. 
Proof. By Assumption 1, $\frac{\partial}{\partial \sigma^{2}} E\left[m(X) \mid \mu, \sigma^{2}\right]$ is non-increasing w.r.t $\sigma^{2}$. Therefore, if $\frac{\partial}{\partial \sigma^{2}} E[m(X) \mid \mu, h(\mu)] \leq 0$, then $\frac{\partial}{\partial \sigma^{2}} E\left[m(X) \mid \mu, \sigma^{2}\right] \leq 0$ for all $\sigma^{2} \geq h(\mu)$. Since Condition 1 implies

$$
\begin{aligned}
\frac{d}{d \mu} E[m(X) \mid \mu, h(\mu)] & \leq 0 \\
\Longleftrightarrow \frac{\partial}{\partial \sigma^{2}} E[m(X) \mid \mu, h(\mu)] h^{\prime}(\mu)+\frac{\partial}{\partial \mu} E[m(X) \mid \mu, h(\mu)] & \leq 0
\end{aligned}
$$

Because $\frac{\partial}{\partial \mu} E[m(X) \mid \mu, h(\mu)] \geq 0$, since it is equivalent to a first-order stochastic dominant shift, and $h^{\prime}(\mu) \geq 0$ by assumption, we must have $\frac{\partial}{\partial \sigma^{2}} E[m(X) \mid \mu, h(\mu)] \leq 0$.

In other words, Condition 1 and Assumption 1 together ensure that the inventor will pick a project with minimum variance $\sigma^{2}$ for a given mean $\mu$, and that the project she picks will have a lower mean $\mu_{M}$ than what the social planner would pick $\mu_{S}$.

\section{$5 \quad$ Policy Interventions}

We have shown that even a risk-neutral inventor will pursue a suboptimal technology if the tradeoff between risk and return rises steeply enough. ${ }^{7}$ We now explore the comparative statics of our model. We then discuss four possible policy interventions: an increase in the technology threshold, a reward for high-value inventions, a tax that disparately penalizes low-value inventions combined with a subsidy that insures against project failure, and a value-based patent system. We also examine how mismeasurement of model parameters by a policymaker might affect policy decisions.

\subsection{Comparative Statics}

In this section, Condition 1 and Assumption 1 are assumed to hold throughout. In terms of our model, we can see how changes to $m, b$, and $\underline{X}$ change the inventor's choice by deriving the following comparative static results for these parameters.

\footnotetext{
${ }^{7}$ Note that throughout this discussion we have not relied on any sort of risk-aversion. Adding that to the analysis would only make the risk/return tradeoff more stark for the inventor, increasing the wedge between the social planner and the inventor.
} 
Proposition 5.1. The inventor's choice of $\mu$ is increasing in $m$.

Proof. Recall the FOC:

$$
-\left(\underline{X}+\frac{b}{m}\right) G_{2}(\underline{X} ; \mu, h(\mu)) h^{\prime}(\mu)-\left(\underline{X}+\frac{b}{m}\right) G_{1}(\underline{X} ; \mu, h(\mu))+\frac{d}{d \mu} \int_{\underline{X}}^{\infty}(x-\underline{X}) d G(x ; \mu, h(\mu))
$$

Differentiating the FOC w.r.t $m$ gives us:

$$
\frac{b}{m^{2}}\left[G_{2}(\underline{X} ; \mu, h(\mu)) h^{\prime}(\mu)+G_{1}(\underline{X} ; \mu, h(\mu))\right]
$$

From Condition 1, we have:

$$
G_{2}(\underline{X} ; \mu, h(\mu)) h^{\prime}(\mu)+G_{1}(\underline{X} ; \mu, h(\mu)) \geq \frac{\frac{d}{d \mu} \int_{\underline{X}}^{\infty}(x-\underline{X}) d G(x ; \mu, h(\mu))}{\left(\underline{X}+\frac{b}{m}\right)}
$$

We know the right hand side term $>0$, which implies quantity $4>0$.

Intuitively, as $m$ increases, the inventor receives more profit than before for each additional unit of social value that she generates. As such, the inventor has greater incentive to produce higher social value inventions.

Put differently, one can view $m \leq 1$ as the proportion of the total welfare generated by the sale of the product that the firm receives. As $m \rightarrow 1$, the firm gains more of the total social welfare and consumer surplus goes to 0 .

Proposition 5.2. The inventor's choice of $\mu$ is decreasing in $b$.

Proof. Differentiating the FOC w.r.t $b$ gives us:

$$
-\frac{1}{m}\left[G_{2}(\underline{X} ; \mu, h(\mu)) h^{\prime}(\mu)+G_{1}(\underline{X} ; \mu, h(\mu))\right]
$$


From Condition 1, we have:

$$
G_{2}(\underline{X} ; \mu, h(\mu)) h^{\prime}(\mu)+G_{1}(\underline{X} ; \mu, h(\mu)) \geq \frac{\frac{d}{d \mu} \int_{\underline{X}}^{\infty}(x-\underline{X}) d G(x ; \mu, h(\mu))}{\left(\underline{X}+\frac{b}{m}\right)}
$$

We know the right hand side term $>0$, which means quantity $5<0$.

As $b$ decreases, the inventor's guaranteed private "boost" from generating any passable invention (i.e., any invention whose social value exceeds $\underline{X}$ ) decreases. This disparately affects lower-value inventions, since both high- and low-value inventions receive the same boost. As such, the private value of high-value inventions relative to low-value ones increases, and the net marginal benefit from increasing $\mu_{M}$ goes up.

What if $\underline{X}$ (i.e., the technology threshold) increases? Differentiating FOC w.r.t $\underline{X}$, we have:

$$
\begin{aligned}
& \frac{d}{d \underline{X}}\left[\frac{d}{d \mu} E[m(X) \mid \mu, h(\mu)]\right]=m \frac{d}{d \underline{X}}\left[\frac{d}{d \mu} \int_{\underline{X}}^{\infty}\left(x+\frac{b}{m}\right) d G(x ; \mu, h(\mu))\right] \\
&=m \frac{d}{d \mu}\left[\frac{d}{d \underline{X}} \int_{\underline{X}}^{\infty}\left(x+\frac{b}{m}\right) d G(x ; \mu, h(\mu))\right]=\frac{d}{d \mu}(m \underline{X}+b) g(\underline{X} ; \mu, h(\mu)) \\
&=(m \underline{X}+b) \frac{d}{d \mu} g(\underline{X} ; \mu, h(\mu))
\end{aligned}
$$

where $g($.$) the pdf of distribution G$. We know that $(m \underline{X}+b)>0$. So the effect of increasing $\underline{X}$ depends on the sign of $\frac{d}{d \mu} g(\underline{X} ; \mu, h(\mu))$.

Proposition 5.3. If $\frac{d}{d \mu} g(\underline{X} ; \mu, h(\mu)) \leq 0$ then the inventor's choice of $\mu$ will be decreasing in $\underline{X}$, and the inventor is more likely to pick a socially-suboptimal innovation as $\underline{X}$ increases. If $\frac{d}{d \mu} g(\underline{X} ; \mu, h(\mu))>0$ then the inventor's choice of $\mu$ will be increasing in $\underline{X}$, and the inventor is less likely to pick a socially-suboptimal innovation as $\underline{X}$ increases.

Proof. If $\frac{d}{d \mu} g(\underline{X} ; \mu, h(\mu)) \leq 0$, then Condition 1 is more likely to hold as $\underline{X}$ increases. If $\frac{d}{d \mu} g(\underline{X} ; \mu, h(\mu))>0$, then Condition 1 is less likely to hold as $\underline{X}$ increases.

So what is the likely sign of $\frac{d}{d \mu} g(\underline{X} ; \mu, h(\mu))$ ? This will of course depend on the underlying distribution $G$. But for many common classes of distributions, the sign is likely to be negative. 
For example, consider the class of exponential distributions. In that case:

$$
\begin{array}{r}
g(\underline{X} ; \mu, h(\mu)) \equiv \frac{1}{\mu} e^{-\frac{X}{\mu}} \\
\Longrightarrow \frac{d}{d \mu} g(\underline{X} ; \mu, h(\mu))=-\frac{1}{\mu^{2}} e^{-\frac{X}{\mu}}+\frac{1}{\mu} \frac{X}{\mu^{2}} e^{-\frac{X}{\mu}}=\frac{e^{-\frac{\underline{X}}{\mu}}}{\mu^{3}}(\underline{X}-\mu)
\end{array}
$$

It is likely that an inventor will pick a project whose expected return $\mu$ exceeds the status quo, $\underline{X}$. As such, quantity (7) will be negative and increasing $\underline{X}$ will make it more likely that Condition 1 holds, thereby increasing the chances an inventor will pick a socially-suboptimal project.

As another example, consider the class of normal distributions:

$$
\begin{array}{r}
g(\underline{X} ; \mu, h(\mu))=\left(\frac{1}{\sqrt{2 \pi h(\mu)}}\right) e^{-\left(\frac{(X-\mu)^{2}}{2 h(\mu)}\right)} \\
\Longrightarrow \frac{d}{d \mu} g(\underline{X} ; \mu, h(\mu))= \\
\left(\frac{1}{\sqrt{2 \pi h(\mu)}}\right) * e^{-\left(\frac{(X-\mu)^{2}}{2 h(\mu)}\right)} *\left((\underline{X}-\mu)\left[\frac{\frac{1}{2} h^{\prime}(\mu)(\underline{X}-\mu)+h(\mu)}{h^{2}(\mu)}\right]\right) \\
+e^{-\left(\frac{(X-\mu)^{2}}{2 h(\mu)}\right)} *\left(-\frac{\pi h^{\prime}(\mu)}{(2 \pi h(\mu))^{3 / 2}}\right) \\
=e^{-\left(\frac{(X-\mu)^{2}}{2 h(\mu)}\right)}\left[(2 \pi h(\mu))^{-1 / 2}\right]\left[(\underline{X}-\mu)\left(\frac{1}{h(\mu)}\right)+\frac{h^{\prime}(\mu)}{2 h(\mu)}\left[\frac{(\underline{X}-\mu)^{2}}{h(\mu)}-1\right]\right]
\end{array}
$$

This implies that $\frac{d}{d \mu} g(\underline{X} ; \mu, h(\mu))<0$ if $h(\mu)>(\underline{X}-\mu)^{2}$ (i.e., the variance at a given $\mu$ increases quickly enough) and $\underline{X}-\mu<0$ (i.e., the expected return $\mu$ exceeds the status quo, $\underline{X}$, as is likely). In that case, quantity (8) will be negative. So once again, increasing $\underline{X}$ will make it more likely that Condition 1 holds, thereby increasing the chances of a socially-suboptimal choice by an inventor. 


\subsection{Increase in Technology Threshold}

We can now explore various policy interventions to align the inventor's incentives with those of the social planner. We begin with a straightforward intervention: an increase in the technology threshold $\underline{X}$.

In some settings, this threshold may depend solely on factors outside a policymaker's control. In the context of pharmaceuticals, however, the FDA may exert considerable control over this level. In particular, drugs whose clinical safety and efficacy profile falls below $\underline{X}$ are not approved and provide zero private returns. Still, while the FDA might be able to increase $\underline{X}$, the existing state of technology in a given field likely sets a floor for this value, because it is unlikely that pharmaceutical companies could obtain significant returns on new drugs that actually perform worse than ones that already exist.

Based on Proposition 5.3 and the discussion in the previous section, we can see it is possible that increasing the technology threshold will increase the chances that an inventor pursues a lower value innovation than the social planner would pursue. The underlying reason is clear: increasing the technology threshold increases the risk that an inventor will obtain zero returns for a given project. As such, even a risk-neutral inventor will pursue a less risky project in such a scenario, if risk increases sharply enough with return. Accordingly, what might seem to be a simple solution - increasing the regulatory technology threshold $\underline{X}$ to deter me-too products may paradoxically cause firms to take on less risk and pursue more me-too innovation.

A corollary of this result is that the problem of suboptimal innovation in a given field might increase as the field advances over time. This is because $\underline{X}$ will continue to increase, and inventors will become more prone to pursuing less risky, socially-suboptimal innovations to reduce their chance of obtaining zero returns.

\subsection{Rewards and Taxes}

Next we derive the following results for a reward or prize system in which the inventor receives a prize $P>0$ if the social value of the invention exceeds some threshold $k$. The optimization 
problem now becomes:

$$
\begin{array}{r}
\max _{\mu, \sigma^{2}} E\left[m(X) \mid \mu, \sigma^{2}, P\right] \\
=\max _{\mu, \sigma^{2}}\left[\int_{\underline{X}}(m x+b) d G\left(x ; \mu, \sigma^{2}\right)+P\left(1-G\left(k ; \mu, \sigma^{2}\right)\right)\right] \\
\Longleftrightarrow \max _{\mu, \sigma^{2}}\left[\int_{\underline{X}}\left(x+\frac{b}{m}\right) d G\left(x ; \mu, \sigma^{2}\right)+\frac{P}{m}\left(1-G\left(k ; \mu, \sigma^{2}\right)\right)\right]
\end{array}
$$

Assumption 2. (Diminishing return to risk) $\frac{\partial^{2}}{\partial\left(\sigma^{2}\right)^{2}} E\left[m(X) \mid \mu, \sigma^{2}, P\right] \leq 0$

Assumption 2 is analogous to Assumption 1; it requires the return to taking more risks at a given level of $\mu$ be decreasing with $\sigma^{2}$ even with the prize system. Similar to the discussion in Section 4 , the sufficient condition for the inventor to pick a suboptimal project now becomes:

\section{Condition 2.}

$$
\begin{array}{r}
\left(\underline{X}+\frac{b}{m}\right) G_{2}(\underline{X} ; \mu, h(\mu)) h^{\prime}(\mu)+\frac{P}{m} G_{2}(k ; \mu, h(\mu)) \geq \\
-\left(\underline{X}+\frac{b}{m}\right) G_{1}(\underline{X} ; \mu, h(\mu))-\frac{P}{m} G_{1}(k ; \mu, h(\mu))
\end{array}
$$

Remark 5.1. When $k=\underline{X}$, any reward $P>0$, is equivalent to an increase in $b$. Thus, such a reward incentivizes the inventor to pick a lower mean project $\mu_{P}<\mu_{M}$.

More generally, a prize that is awarded for crossing too low of a threshold could be counterproductive: it might encourage that inventor to actually produce a lower-value invention.

Is there a situation under which the reward system helps mitigate the problem? Since $-\frac{P}{m} G_{1}(k ; \mu, h(\mu))>0$, Condition 2 will be less likely to be satisfied if $G_{2}(k ; \mu, h(\mu))<0$.

Proposition 5.4. Suppose the inventor, whose status quo choice is $\mu_{M}$, receives a prize $P>0$ if the social value of the invention exceeds some threshold $k$. If his expected return exhibits diminishing return to risk (Assumption 2) and Condition 2 holds, then the inventor's choice $\mu_{P}$ is suboptimal. Furthermore, if $G_{2}(k ; \mu, h(\mu))<0$, he will select a project with $\mu_{P}>\mu_{M}$.

Proof. Follows from the discussion above. 
We can see what is the minimal level at which a prize will improve inventor incentives for the class of normal distributions. In that case:

$$
\begin{array}{r}
G(k ; \mu, h(\mu))=\frac{1}{2}\left[1+\operatorname{erf}\left(\frac{k-\mu}{\sqrt{2 h(\mu)}}\right)\right] \\
\Longrightarrow G_{2}(k ; \mu, h(\mu))=\frac{1}{2} \frac{2}{\sqrt{\pi}} e^{-\left(\frac{k-\mu}{\sqrt{2 h(\mu)}}\right)^{2} \frac{k-\mu}{\sqrt{2}} \frac{-1}{2} h(\mu)^{-\frac{3}{2}}} \\
=-\frac{1}{2 \sqrt{2 \pi}} e^{-\left(\frac{k-\mu}{\sqrt{2 h(\mu)}}\right)^{2}} \frac{k-\mu}{\sqrt{2} h(\mu)^{-\frac{3}{2}}}
\end{array}
$$

Therefore, $G_{2}(k ; \mu, h(\mu))<0$ if $k>\mu$. Again, the intuition here is straightforward: if a prize or reward is provided for inventions whose social value exceeds a sufficiently high threshold (i.e., $k \geq \mu$ ), then the inventor will select an investment project with higher unconditional expected social returns than in the absence of a prize.

Given that low-end rewards are problematic, a lump-sum tax might reduce the gap between the inventor and the social planner. In fact, we can easily derive the following result:

Corollary 5.1. If Condition 1 and Assumption 1 hold, an inventor-specific lump sum tax of $\tau=b$ placed on all innovation that exceeds the technology threshold $\underline{X}$ will help align the inventor's incentives with those of the social planner.

Proof. Such a tax is identical to a corresponding reduction in $b$. Note that this disproportionately affects lower-value inventions that exceed the technology threshold $\underline{X}$.

Corollary 5.2. If Condition 1 and Assumption 1 hold, a subsidy $s=X$ for innovations lower than the technology threshold $\underline{X}$ and $s=(1-m) X$ for innovations higher than $\underline{X}$ together with a inventor-specific lump sum tax will fully align the inventor's incentives with those of the planner.

Proof. The combination of the subsidy and the tax will align the inventor's return function with that of the social planner.

The subsidy below $\underline{X}$ acts as insurance for projects that fall below the technology threshold, thereby encouraging the inventor to take on more risk. The subsidy above $\underline{X}$ acts like a value- 
based patent system, discussed in more detail below. In particular, since $m \leq 1$, this operates as a subsidy that disparately benefits higher-value inventions. ${ }^{8}$

\subsection{Value-Based Patent Protection}

Finally, what about changes to the patent system? Patents give inventors a legal monopoly over their inventions, enabling them to exclude others from making, using, selling, offering to sell, or importing their inventions into a country for a limited period of time. Whether this legal monopoly translates into an economic monopoly depends on market demand. All else being equal, we would expect a patent on a product that generates more social value to generate higher profits. That relationship is captured by the profit function in our model, with $m>0$.

Ideally, we want a system where the increased benefit to the inventor is proportional to the social value of the invention. This sort of value-based patent system would boost profits for inventions with higher social value as compared to those that have lower social value. As a practical matter, this might be implemented by adjusting patent duration based on the social value of the underlying invention. In terms of our model, we can think about the effect of increased patent protection as an strict increase in the slope parameter $m$.

Proposition 5.5. Suppose the inventor receives an increase in patent protection proportional to the invention's underlying social value, which is equivalent to an increase from $m$ to $m^{\prime}>m$. Under Assumption 1, the inventor will pick $\mu_{M}^{\prime}>\mu_{M}$.

Proof. This follows directly from Proposition 5.1.

Note that, if in addition to increasing $m$, we decrease patent protection for low-value inventions, that would be equivalent to lowering $b$. From Proposition 5.2 above, we can see this would also increase an inventor's choice of $\mu$.

Instead of a value-based patent system, why not more just increase patent protection uniformly across the board, regardless of the social value of the invention? Such a increase would provide a boost to all products that generate positive profits for a firm (i.e., where $X>\underline{X}$ ). In

\footnotetext{
${ }^{8}$ By contrast, one can see that a proportional tax - one that takes a fixed percentage of pharmaceutical profits - might exacerbate firms' tendency to pick low-value inventions. This follows directly from Proposition 5.1, since a proportional tax in effect reduces the slope $m$ of the inventor's profit function.
} 
our model, this would be roughly modeled as an increase in the intercept parameter, $b$. But as we have seen, such a boost would actually increase the gap between the inventor's and social planner's incentives. To the extent we want to minimize deviations from the status quo, such a policy would be suboptimal, because we would need to increase the slope parameter $m$ even more to counteract the effect of raising $b$ at the same time.

\subsection{Policy under Incomplete Information}

Throughout this paper, we have assumed the policymaker has the same complete information as the representative firm. But if the firm has private unobservable information in its investment choice set, then the policymaker may have a biased estimate of model parameters, which could lead to wasteful policy interventions such as unnecessary or excessive rewards to induce innovation.

To illustrate, consider a reward intervention in the monopoly discrete choice model as discussed in Section 3.1 above. Let the social cost of raising money for the reward be a continuous function $S(r)$, where $S^{\prime}(r) \geq 0$. This social cost may be, for example, the deadweight loss caused by taxation used to raise revenues to pay the reward. Assume as before $p_{H} \bar{X}_{H}-p_{L} \bar{X}_{L}>0$. The social planner does not know $p_{H}$ but has an estimate $\hat{p}_{H}$. It knows the other model parameters $\bar{X}_{H}, p_{L}, \bar{X}_{L}, \underline{X}, b$.

Proposition 5.6. The only situation in which a reward system might be worse than the status quo is if the social planner underestimates $p_{H}$.

Proof. In Appendix.

In the worst-case situation, the firm is already picking the higher-value project, and the planner incorrectly perceives the firm as picking the lower-value project. Accordingly, the planner would offer a windfall, or a reward that was unnecessary to induce the higher-value choice.

For other model parameters, $\bar{X}_{H}, p_{L}, \bar{X}_{L}, b,{ }^{9}$ similar logic applies. In particular, a reward system might be worse than the status quo only if the social planner underestimates $\bar{X}_{H}$ or

\footnotetext{
${ }^{9}$ We assume $\underline{X}$ always yields zero payoffs; this can be viewed as a normalization and hence any mismeasurement of $\underline{X}$ is captured by mismeasurement of $\bar{X}_{H}$ and $\bar{X}_{L}$.
} 
overestimates $p_{L}, \bar{X}_{L}$, or $b$. In these scenarios, the firm would receive a windfall, since it would have chosen the high-value project even if no reward had been provided.

The potential for mismeasurement identified here is unlikely to lead to outcomes worse than the status quo. If anything, we might worry about overly optimistic social planners who overestimate $p_{H}$ and/or $\bar{X}$ (i.e., they believe the chances of success or the value of the highvalue project are greater than they actually are) or underestimate $p_{L}$ and/or $\bar{X}_{L}$ (i.e., they believe the chances of success and value of the lower-value project are less than they actually are). These measurement errors would, at worst, lead to the status quo. While overestimates of $b$ might cause policymakers to provide windfall payments to firms, this parameter is actually quite measurable - for example, as discussed in the context of metastatic colorectal cancer drugs, we can estimate $b$ by measuring price markups for new drugs that marginally improve over inexpensive treatments like chemotherapy. As such, mismeasurement probably should not be a significant concern for policymakers and should not deter them from implementing the policy interventions proposed here.

\section{Conclusion}

Our paper models how risk and return influence the investments that technology companies make when investing in new products. In particular, we show if firms obtain a fixed private benefit from producing new technologies that marginally exceed the state of the art, firms will invest in less risky, socially-suboptimal R\&D projects. We further show that competition and new entrants mitigate but do not eliminate the distortion created by the fixed private benefit. For pharmaceuticals and biotechnology firms, our model formalizes a motivation for these firms to "reach for mediocrity" and pursue me-too innovations - less risky projects that add little social benefit over the status quo.

We also explore various policy interventions that could be used to reduce the gap in incentives between inventors and the social planner. We show that an increase in the technology threshold is ambiguous in this regard, though it may exacerbate the problem we identify here for common classes of return distributions. By contrast, rewards for high-value inventions and lump-sum taxes that disparately affect low-value inventions will reduce the potential problem. 
In addition, we show that a value-based patent system, with increasing patent rents provided to inventors based on the underlying social value of the invention, could induce inventors to pursue optimal risk/return tradeoffs and innovations that are maximally socially beneficial. We explain why such a system would be more effective than across the board increases in patent protection.

Since the wedge between private and social incentives appears to be especially large in the context of pharmaceuticals, this would be an obvious place where a value-based patent system could be useful. How might this system be implemented in practice? As we discuss in our related work (Sukhatme and Bloche [2018]), there are a number of existing metrics of clinical safety and efficacy that enable us to measure drug value. For instance, quality-added life years are an increasingly commonly-used metric that measures average increases in lifespan provided by drugs, discounted by their effect on patients' quality of life. And in the context of cancer medication, the American Society of Clinical Oncology has recently suggested value-based metrics for patients and doctors to consider when choosing therapies (Schnipper et al. [2015]). Such metrics could be adapted to form the basis of a value-based drug patent system. This approach would enable significant innovations to receive greater rewards than me-too products, thereby channeling private resources toward more socially-beneficial outcomes. 


\section{Appendix}

\section{A Proof of Proposition 5.6}

Proof. It is socially optimal to offer a reward $r$ only within the range $0<r_{\min }<r \leq r_{\max }$, where these values are:

$$
\begin{array}{r}
r_{\text {min }}=\max \left[\frac{p_{L}}{p_{H}}\left(\bar{X}_{L}+b\right)-\left(\bar{X}_{H}+b\right), 0\right] \\
r_{\text {max }}=S^{-1}\left(\bar{X}_{H}-\frac{p_{L}}{p_{H}} \bar{X}_{L}\right)
\end{array}
$$

If $r_{\min }>r_{\max }$, then the benefit from inducing the higher-value project is less than the social cost of generating the reward. If $r_{\min } \leq r_{\max }$, then the socially-optimal reward is $r_{\text {min }}$. Note that if $\frac{p_{L}}{p_{H}}\left(\bar{X}_{L}+b\right)-\left(\bar{X}_{H}+b\right)<0$, the reward is 0 because the firm is already picking the higher-value project.

Now suppose the planner observes $p_{H}$ with error, such that $\hat{p}_{H}<p_{H}$. Then the planner's perceived $\left(\hat{r}_{\min }, \hat{r}_{\text {max }}\right)$ will be:

$$
\begin{array}{r}
\hat{r}_{\text {min }}=\max \left[\frac{p_{L}}{\hat{p}_{H}}\left(\bar{X}_{L}+b\right)-\left(\bar{X}_{H}+b\right), 0\right] \geq r_{\text {min }} \\
\hat{r}_{\max }=S^{-1}\left(\bar{X}_{H}-\frac{p_{L}}{\hat{p}_{H}} \bar{X}_{L}\right)<r_{\max }
\end{array}
$$

Note that if $r_{\min }>r_{\max }$ then $\hat{r}_{\min }>\hat{r}_{\max }$. So the planner will correctly eschew providing a reward if the social costs of the reward exceed the benefits. If $\hat{r}_{\min }>\hat{r}_{\max }$ but $r_{\min } \leq r_{\max }$, then the planner will not provide a reward even though doing so would be socially optimal, and the status quo will remain.

Now examine two cases - first, assume the firm picks the lower-value project, so we know $p_{L}\left(\bar{X}_{L}+b\right)>p_{H}\left(\bar{X}_{H}+b\right)$ and so $r_{\min }>0$. The social planner will correctly infer the firm is picking the lower-value project, since $\hat{r}_{\text {min }} \geq r_{\text {min }}>0$. The planner will offer to pay more 
than the optimal reward $r_{m i n}$, but would not do worse than the status quo, where no reward is provided.

Next, let $p_{L}\left(\bar{X}_{L}+b\right)<p_{H}\left(\bar{X}_{H}+b\right)$ and so $r_{\text {min }}=0$; accordingly, the firm is already picking the higher-value project. If $p_{L}\left(\bar{X}_{L}+b\right)<\hat{p}_{H}\left(\bar{X}_{H}+b\right)$, then the planner will still correctly infer the firm's choice and will not offer a reward. If $\hat{p}_{H}$ is low enough such that $p_{L}\left(\bar{X}_{L}+b\right)>\hat{p}_{H}\left(\bar{X}_{H}+b\right)$, the planner will incorrectly believe the firm is picking the lowervalue project and will offer $\hat{r}_{\min }$ as a reward, so long as $\hat{r}_{\min } \leq \hat{r}_{\max }$. This will generate an unnecessary social cost $S\left(\hat{r}_{m i n}\right)$.

Now suppose instead $\hat{p}_{H}>p_{H}$. Then the perceived $\left(\hat{r}_{\min }, \hat{r}_{\max }\right)$ will be:

$$
\begin{array}{r}
\hat{r}_{\text {min }}=\max \left[\frac{p_{L}}{\hat{p}_{H}}\left(\bar{X}_{L}+b\right)-\left(\bar{X}_{H}+b\right), 0\right] \leq r_{\text {min }} \\
\hat{r}_{\text {max }}=S^{-1}\left(\bar{X}_{H}-\frac{p_{L}}{\hat{p}_{H}} \bar{X}_{L}\right)>r_{\text {max }}
\end{array}
$$

First, assume $p_{L}\left(\bar{X}_{L}+b\right)>p_{H}\left(\bar{X}_{H}+b\right)$, so the firm chooses the lower-value project. If $p_{L}\left(\bar{X}_{L}+b\right)<\hat{p}_{H}\left(\bar{X}_{H}+b\right)$, then the planner will mistakenly believe the firm is already picking the higher-value project and will keep the status quo. If $p_{L}\left(\bar{X}_{L}+b\right)>\hat{p}_{H}\left(\bar{X}_{H}+b\right)$, then the planner will offer to pay the reward $\hat{r}_{\text {min }}$, so long as $\hat{r}_{\text {min }}>0$ and $\hat{r}_{\text {min }} \leq \hat{r}_{\text {max }}$. But since $\hat{r}_{\min }<r_{\min }$, the reward would be insufficient to induce the firm to pick the higher-value project, and the status quo will remain.

Now instead suppose $p_{L}\left(\bar{X}_{L}+b\right)<p_{H}\left(\bar{X}_{H}+b\right)$ or $r_{m i n}=0$, so the firm is already picking the higher-value project. Since $\hat{r}_{\min } \leq r_{\min }=0$, it must be that $\hat{r}_{\min }=0$. The planner will correctly infer the firm's choice and will not offer a reward. 


\section{References}

Fernando Antoñanzas, Carmelo Juárez-Castelló, and Roberto Rodríguez-Ibeas. Innovation, loyalty and generic competition in pharmaceutical markets. SERIEs: Journal of the Spanish Economic Association, 2(1):75-95, 2011.

David Bardey, Antoine Bommier, and Bruno Jullien. Retail price regulation and innovation: reference pricing in the pharmaceutical industry. Journal of Health Economics, 29(2):303-316, 2010.

Joseph A DiMasi and Cherie Paquette. The economics of follow-on drug research and development: Trends in entry rates and the timing of development. Pharmacoeconomics, 23(12): 1193-1202, 2005.

Mats Ekelund and Björn Persson. Pharmaceutical pricing in a regulated market. the Review of Economics and Statistics, 85(2):298-306, 2003.

Tito Fojo, Sham Mailankody, and Andrew Lo. Unintended consequences of expensive cancer therapeutics; the pursuit of marginal indications and a me-too mentality that stifles innovation and creativity: the john conley lecture. JAMA Otolaryngology-Head \& Neck Surgery, 140(12): $1225-1236,2014$.

Juan-José Ganuza, Gerard Llobet, and Beatriz Domínguez. R\&d in the pharmaceutical industry: a world of small innovations. Management Science, 55(4):539-551, 2009.

Paula González, Ines Macho-Stadler, and David Pérez-Castrillo. Private versus social incentives for pharmaceutical innovation. Journal of health economics, 50:286-297, 2016.

Henry G Grabowski, Joseph A DiMasi, and Genia Long. The roles of patents and research and development incentives in biopharmaceutical innovation. Health Affairs, 34(2):302-310, 2015.

Daniel Jacob Hemel and Lisa Larrimore Ouellette. Beyond the patents-prizes debate. 2013.

Arjun Jayadev and Joseph Stiglitz. Two ideas to increase innovation and reduce pharmaceutical costs and prices. Health Affairs, 28(1):w165-w168, 2009. 
Michael Kremer. Patent buyouts: A mechanism for encouraging innovation. The Quarterly Journal of Economics, 113(4):1137-1167, 1998.

Joshua Krieger, Danielle Li, and Dimitris Papanikolaou. Developing novel drugs. 2018.

Chris P Lee, Glenn M Chertow, and Stefanos A Zenios. An empiric estimate of the value of life: updating the renal dialysis cost-effectiveness standard. Value in Health, 12(1):80-87, 2009.

Patrick Leoni and Alvaro Sandroni. Can patent duration hinder medical innovation. International Journal of Health Economics and Management, 16(4):397-406, 2016.

Z John Lu and William S Comanor. Strategic pricing of new pharmaceuticals. The Review of Economics and Statistics, 80(1):108-118, 1998.

Stephane Régnier. What is the value of 'me-too' drugs? Health Care Management Science, 16(4):300-313, 2013. ISSN 1572-9389. doi: 10.1007/s10729-013-9225-3. URL http://dx.doi.org/10.1007/s10729-013-9225-3.

Susan Rose-Ackerman. Risk taking and ruin: Bankruptcy and investment choice. The Journal of Legal Studies, 20(2):277-310, 1991. ISSN 00472530, 15375366. URL http://www.jstor.org/stable/724441.

Lowell E Schnipper, Nancy E Davidson, Dana S Wollins, Courtney Tyne, Douglas W Blayney, Diane Blum, Adam P Dicker, Patricia A Ganz, J Russell Hoverman, Robert Langdon, et al. American society of clinical oncology statement: a conceptual framework to assess the value of cancer treatment options. Journal of Clinical Oncology, 33(23):2563-2577, 2015.

Steven Shavell and Tanguy Van Ypersele. Rewards versus intellectual property rights. The Journal of Law and Economics, 44(2):525-547, 2001.

Neel U. Sukhatme and M. Gregg Bloche. Solving the enigma of health care costs: Dynamic moral hazard and value-based patents. Working paper (on file with author), 2018. 